ANNALES

POLONICI MATHEMATICI

XLVIII (1988)

\title{
Asymptotic behaviour of solutions of the nonlinear heat equation
}

\author{
by Tomasz Drotko (Katowice)
}

\begin{abstract}
The equation arising in biology is studied. Using the method of differential inequalities and Lapunov functions, we describe the behaviour of solutions of (1)-(3) as $t \rightarrow \infty$. Usually the solutions tend to one of the stationary solutions 0 or 1 . In the end of the work some remarks concerning global in time existence of solutions and a priori estimates are presented.
\end{abstract}

1. Introduction. The present paper is stimulated by the recent result of Anderson [1] concerning the differential equation

$$
u_{1}=u_{x x}+f(x, u)
$$

in the set $D:=\left\{(t, x) \in R^{2} ; t>0, x \in(0, a)\right\}$, with additional conditions

$$
\begin{array}{ll}
u_{x}(t, 0)=u_{x}(t, a)=0 & \text { for } t>0, \\
u(0, x)=\varphi(x) & \text { for } x \in(0, a)
\end{array}
$$

and nonlinear function $f$. This equation admits interesting biological interpretation (as in [1]). Suppose, namely, that two competing allospecies $X$ and $Y$ share a breeding range which can be represented as a one-dimensional interval of space $0 \leqslant x \leqslant a$. Suppose that $X$ and $Y$ are presented at location $x$ and time $t$ in proportions $u$ : $(1-u)$, where $u$ is a function of $x$ and $t$ and satisfies $0 \leqslant u(t, x) \leqslant 1$. Then the model of the competitive interaction of species $X$ and $Y$ is given by (1)-(3). The function $f$ represents the dynamics of the local competitive interaction.

The behaviour of solutions of (1)-(3) depends heavily upon the initial function $\varphi$, thus it is interesting to determine the regions of stability in dependence on $\varphi$ and $f$.

Definitions. We say that $w$ is a stationary solution of problem (1)-(3) if $w$ fulfils the Neumann problem:

$$
\begin{gathered}
w_{x x}+f(x, w)=0 \quad \text { for } x \in(0, a), \\
w_{x}(0)=w_{x}(a)=0 .
\end{gathered}
$$


We consider the following real Banach spaces: $C^{0}([0, a]), C^{2+a}([0, a])$, $C^{1+\alpha / 2,2+\alpha}(\bar{D})$ (see [6]), $L^{2}(0, a)$.

We need also the special versions of the Poincare inequality (see [9]):

$$
\underset{c>0}{\exists} \underset{v \in C_{0}^{1}([0 . a))}{\forall}\|v\|_{L^{2}}^{2} \leqslant c\left\|v_{x}\right\|_{L^{2}}^{2},
$$

where $C_{0}^{1}$ denotes the space of all $C^{1}$ functions vanishing for $x=0$ and $x=a, L^{2}=L^{2}(0, a)$,

$$
\underset{C>0}{\exists} \underset{v \in C^{1}([0, a])}{\forall}\|v-\bar{v}\|_{L^{2}}^{2} \leqslant C\left\|v_{x}\right\|_{L^{2}}^{2},
$$

where $\bar{v}=a^{-1} \int_{0}^{a} v(z) d z$.

2. Assumptions. Classical solutions of the problem (1)-(3) are investigated. Existence (local in time) of solutions of such problems is shown in [7], [6], see also Section 7 below.

Let $f$ and $\varphi$ satisfy the conditions:

(A) The function $f:[0, a] \times[0,1] \rightarrow R$ belongs to $C^{1}([0, a] \times[0,1])$ and, for any fixed $x \in[0, a], f$ has' three different roots $0, \lambda_{x}, 1$ such that $0<\lambda_{x}<1$. The value $f(x, z)$ is strictly negative for $z \in\left(0, \lambda_{x}\right)$, and strictly positive for $z \in\left(\lambda_{x}, 1\right)$.

(B) The function $f:[0,1] \rightarrow R$ belongs to $C^{2+a}([0,1])$, and has three different roots $0<\lambda<1$; its value $f(z)$ is strictly negative for $z \in(0, \lambda)$ and strictly positive for $z \in(\lambda, 1)$.

(C) The function $\varphi:[0, a] \rightarrow[0,1]$ belongs to $C^{2+\alpha}([0, a])$ and satisfies the compatibility condition

$$
\varphi_{x}(0)=\varphi_{x}(a)=0 .
$$

Such $\varphi$ is called the initial condition.

3. Preliminaries. We need a special version of Theorem 64.3 of [11]:

Proposition 1. Let the functions $u$ and $w$ satisfy the differential inequalities

$$
\begin{aligned}
& u_{1} \leqslant u_{x x}+f(x, u) \\
& w_{1} \geqslant w_{x x}+f(x, w)
\end{aligned} \text { in } D,
$$

together with the conditions

$$
\begin{gathered}
u(0, x)=\varphi(x), \quad w(0, x)=\psi(x) \quad \text { for } x \in[0, a], \\
u_{x}(t, 0)=u_{x}(t, a)=0=w_{x}(t, 0)=w_{x}(t, a) \quad \text { for } t>0 .
\end{gathered}
$$


Let the function $f$ be continuous in $(x, u)$ and Lipschitz with respect to the second argument:

$$
\underset{c>0}{\exists} \underset{h, l \in[0,1]}{\forall} \underset{x \in[0, a]}{\forall}|f(x, h)-f(x, l)| \leqslant c|h-l| .
$$

If $\varphi(x) \leqslant \psi(x)$ for $x \in[0, a]$, then the classical solutions satisfy $u(t, x)$ $\leqslant w(t, x)$ in $D$.

Proposition 1 implies the following corollaries:

Corollary 1. Let the function $f$ satisfy

$$
\underset{i>0}{\exists} \underset{x \in[0, a]}{\forall} \underset{: \leqslant \lambda}{\forall} f(x, z) \leqslant 0,
$$

and let, for the initial condition $\varphi, 0 \leqslant \varphi(x) \leqslant m \leqslant \lambda$ holds. Then the solution $u$ of (1)-(3) is bounded from above by $m$.

Corollary 2. Uniqueness of the solution of problem (1)-(3) under assumption (A) is an easy consequence of Proposition 1.

COROllary 3. If the solution $u$ corresponding to the initial condition $\varphi$ tends to zero as $t$ tends to infinity, then any solution corresponding to an initial condition $\psi$ such that $\psi(x) \leqslant \varphi(x), x \in[0, a]$, also tends to zero.

The next lemma describes the behaviour of the linear heat equation at infinity, the special case of the fundamental theorem of [10].

LEMMA 1. All the solutions of the heat equation

$$
u_{t}=u_{x x}, \quad u_{x}(t, 0)=u_{x}(t, a)=0, \quad u(0, x)=\varphi(x)
$$

converge to $\bar{\varphi}=a^{-1} \int_{0}^{a} \varphi(x) d x$ uniformly in $[0, a]$.

Proof. Integrating the equation over $[0, a]$, we verify that the average $\bar{u}(t)=a^{-1} \int_{0}^{a} u(t, x) d x$ is constant in time and equal to $\bar{\varphi}$. Also the function $U(t, x):=u(t, x)-\bar{\varphi}$ is given by the Fourier series

$$
U(t, x)=\sum_{n=0}^{\infty} c_{n} \cos \left(\frac{n \pi}{a} x\right) \exp \left(-\frac{n^{2} \pi^{2}}{a^{2}} t\right)
$$

with

$$
c_{n}=2 a^{-1} \prod_{0}^{a}(\varphi(x)-\bar{\varphi}) \cos \left(\frac{n \pi}{a} x\right) d x, \quad n=1,2, \ldots, \quad c_{0}=0 .
$$

Hence $(u(t, \cdot)-\bar{\varphi})$ tends to 0 as $t \rightarrow \infty$. 
4. The method of differential inequalities. We shall consider stability of stationary solutions 0 and 1 of the problem

$$
\begin{gathered}
u_{t}=u_{x x}+f(u) \quad \text { in } D, \\
u_{x}(t, 0)=u_{x}(t, a)=0, \quad t>0, \\
u(0, x)=\varphi(x), \quad x \in[0, a] .
\end{gathered}
$$

Since the properties of $f(u)$ in the interval $[0, \lambda]$ are analogous to the properties of $-f(1-u)$ in the interval $[\lambda, 1]$, we can restrict our studies to the first interval and the convergence of the solutions to zero.

THEOREM 1. Let the functions $f$ and $\varphi$ satisfy conditions (B), (C); additionally, let $f^{\prime}\left(0^{+}\right)<0$ and

$$
0 \leqslant \varphi(x) \leqslant \lambda, \quad \bar{\varphi}<\lambda .
$$

Then the solution $u$ of (8)-(10) tends to 0 as $t \rightarrow \infty$ uniformly in $[0, a]$.

Proof. The proof consists of two parts. First we shall prove that for $t$ sufficiently large $u(t, x)<\lambda$. Consider the comparison system

$$
w_{t}=w_{x x}, \quad w_{x}(t, 0)=w_{x}(t, a)=0, \quad w(0, x)=\varphi(x) .
$$

Since $0 \leqslant \varphi(x) \leqslant \lambda$, by Proposition 1 we have $0 \leqslant w(t, x) \leqslant \lambda$; hence from condition (B) $f(w(t, x)) \leqslant 0$ and

$$
w_{t} \geqslant w_{x x}+f(w) \text {. }
$$

Applying Proposition 1 to $u$ and $w$, we get

$$
u(t, x) \leqslant w(t, x) \quad \text { in } D .
$$

Since by Lemma $1 w$ tends to $\bar{\varphi}$, then for $T_{0}>0$

$$
\underset{\varepsilon>0}{\exists} \underset{t \geqslant T_{0}>0}{\forall} w(t, x) \leqslant \bar{\varphi}+\varepsilon \leqslant \lambda-\varepsilon
$$

and, moreover, $u(t, x) \leqslant \lambda-\varepsilon$ for $t \geqslant T_{0}$.

Next we show that $u$ converges to zero. The new comparison system in the cylinder $D_{T_{0}}:=D \cap\left\{t>T_{0}\right\}$ is considered. Initial condition is given for $t=T_{0}$ :

$$
\begin{aligned}
u_{t} & =u_{x x}+f(u) & & \text { in } D_{T_{0}}, \\
u_{x}(t, 0) & =u_{x}(t, a)=0 & & \text { for } t>T_{0}, \\
u\left(T_{0}, x\right) & =\varphi_{1}(x) & & \text { for } x \in[0, a],
\end{aligned}
$$

where $\varphi_{1}$ is the value of the solution of (8)-(10) for $t=T_{0}$. We have $0 \leqslant \varphi_{1}(x) \leqslant \lambda-\varepsilon$, and by Corollary 1 , for $t \geqslant T_{0}$

$$
0 \leqslant u(t, x) \leqslant \max _{[0, a]} \varphi_{1}(x) \leqslant \lambda-\varepsilon .
$$


Condition $f^{\prime}\left(0^{+}\right)<0$ and strict negativity of $f$ on the interval $(0, \lambda)$ imply that

$$
\underset{\Omega>0}{\exists} \underset{z \in[0, \lambda-\varepsilon]}{\forall} f(z) \leqslant-\varrho z,
$$

and, in particular, since $0 \leqslant u(t, x) \leqslant \lambda-\varepsilon ; t \geqslant T_{0}$, we have

$$
f(u(t, x)) \leqslant-\varrho u(t, x) \quad \text { for } t \geqslant T_{0} .
$$

Consider together with (12)-(14) the comparison problem

$$
v_{t}=v_{x x}-\varrho v \quad \text { in } D_{T_{0}}
$$

with conditions (13)-(14) for $v$. Proposition 1 gives $0 \leqslant u(t, x) \leqslant v(t, x)$, and since the transformed function $\eta(t, x):=v(t, x) \exp \left(\varrho\left(t-T_{0}\right)\right)$ satisfies the linear heat equation (and hence is bounded), we verify that

$$
0 \leqslant v(t, x)=\eta(t, x) \exp \left(-\varrho\left(t-T_{0}\right)\right) \rightarrow 0, \quad t \rightarrow \infty
$$

uniformly in $[0, a]$.

Remark 1. Theorem 1 remains true under assumptions (A), (C) with $f$ depending on $x$ if the value $\varrho$ in (15) will be chosen common for $x \in[0, a]$.

5. The combined method. Theorem 1 gives information about the behaviour of solutions only when $0 \leqslant \varphi(x) \leqslant \lambda$ or $\lambda \leqslant \varphi(x) \leqslant 1$. The initial function $\varphi$ could not intersect the value $\lambda$. We want to study now possible limit states of solutions. This will be done with the use of the concept of Lapunov functions. As was shown by Chafee [2], all possible limit states of trajectories are contained in the set of stationary solutions of (8)-(10). We want to study some special cases of this convergence.

As a consequence of (B), (C) the solution $u$ of (8)-(10) is smooth; its derivatives $D_{x}^{4} u, D_{1} D_{x}^{2} u$ are Hölder continuous (exponent $\alpha$ ) in any cylinder $[\gamma, T] \times[0, a]$ with $\gamma>0$. Moreover, we have (see Section 7 ) global in time estimates of the derivatives of $u$ in $C^{0}([0, a])$ :

$$
\left\|u_{x}(t, \cdot)\right\|_{C^{0}} \leqslant \text { const, } \quad\left\|u_{x x}(t, \cdot)\right\|_{C^{0}} \leqslant \text { const, } \quad\left\|u_{t}(t, \cdot)\right\|_{C^{0}} \leqslant \text { const } .
$$

Hence, as a consequence of the Ascoli-Arzela theorem, the trajectorie $\{u(t, \cdot)\}_{t \geqslant 0}$ is compact in $C^{1}([0, a])$ phase space; for any sequence $\left\{t_{n}\right\}_{n \in N}$, $t_{n} \rightarrow \infty$, we can extract a subsequence such that for some $v \in C^{1}([0, a])$

$$
u\left(t_{n_{k}}, \cdot\right) \rightarrow v \quad \text { in } C^{1}([0, a]) .
$$

We can study now stability of the constant stationary solutions $0, \lambda, 1$.

THEOREM 2. Let conditions (B), (C) hold and let $u$ be a solution of (8)-(10) such that

$$
\underset{t \geqslant 0}{\forall} \int_{0}^{a} f(u(t, x)) u(t, x) d x \leqslant 0
$$


Then any sequence $i u\left(t_{n}, \cdot\right) !, t_{n} \rightarrow x$, convergent in $C^{1}([0, a])$ tends to one of the limits $0, i$ or 1.

Proof. Multiplying (8) by $u$ and integrating over $[0, a]$, we get $\left(I^{2}=L^{2}(0, a)\right)$

$$
\frac{d}{d t}\|u(t, \cdot)\|_{L^{2}}^{2}=-2 \prod_{0}^{a}\left(u_{x}\right)^{2} d x+2 \prod_{0}^{a} f(u) u d x
$$

Now the quantity $L \Phi:=\|\Phi\|_{L^{2}}^{2}$ will play a role of the Lapunov function (see [8]) for our problem. More precisely, $L$ is a Lapunov function on the closed subset of $\mathbb{C}^{1}([0, a])$

$$
C:=\left\{\Phi \in C^{1}([0, a]): \int_{0}^{a} f(\Phi(x)) \Phi(x) d x \leqslant 0, \Phi_{x}(0)=\Phi_{x}(a)=0\right\} .
$$

The derivative of $L$ along the solution $u$ satisfying (18) is non-positive; hence as a result of the general theory [8] the set of possible limit states consists of such functions $v$ belonging to $C$, for which the right-hand side of (19) vanishes. In particular,

$$
\int_{0}^{a}\left(v_{x}\right)^{2} d x=0
$$

which is possible only for $v(x) \equiv$ const $=c$. Now, since also the second component must vanish, we have

$$
\int_{0}^{a} f(c) c d x=\operatorname{acf}(c)=0
$$

which is possible only for $c$ equal to $0, \lambda$ or 1 .

Next theorem shows realizations of (generally implicit) assumption (18) of Theorem 2.

THEOREM 3. Let $f$ be as in Theorem 1. Then for every positive $\varepsilon \leqslant \lambda$ there exists a $\delta(\varepsilon)>0$, such that if

$$
a^{-1} \prod_{0}^{a} \varphi(x) d x=\bar{\varphi}<\lambda-\varepsilon \quad \text { and } \quad 0 \leqslant \varphi(x)<\lambda+\delta(\varepsilon),
$$

then corresponding to the initial condition $\varphi$ solution $u$ of the problem (8)-(10) tends uniformly to zero.

Proof. If $0 \leqslant \varphi(x) \leqslant \lambda$, then by Corollary 1 for all $t \geqslant 0$ we conclude that $0 \leqslant u(t, x) \leqslant \lambda$, and hence, as a consequence of Theorem $1, u$ tends to zero.

Consider the general case. Let the initial condition $\varphi$ satisfy our 
assumptions (with $\delta(\varepsilon)$ which will be determined later) and let $\psi(x):=\min : \varphi(x) ; \lambda !$. Writing $\gamma:=\max _{[0, a]}(\varphi(x)-\psi(x))$, we have

$$
\varphi(x) \leqslant i+\gamma, \quad 0 \leqslant \varphi(x)-\psi(x) \leqslant \gamma .
$$

However, the function $\psi$ is only continuous and satisfies (which is easy to check) $\psi^{\prime}\left(0^{+}\right)=\psi^{\prime}\left(a^{-}\right)=0$; it will be taken as the initial function, moreover, Proposition 1 works also for such initial functions. Consider the comparison system for the problem (8)-(10):

$$
v_{t}=v_{x x}, \quad v_{x}(t, 0)=v_{x}(t, a)=0, \quad v(0, x)=\psi(x) .
$$

Proposition 1 and Lemma 1 used for $v$ and the solution $w$ of the problem (8)-(9) with the initial function $\psi$ give:

$$
\underset{T_{0}>0}{\exists} \underset{t \geqslant T_{0}}{\forall} w(t, x) \leqslant v(t, x)<\lambda-\frac{1}{2} \varepsilon .
$$

Moreover, $T_{0}$ depends on $\varepsilon$ only, because it will be find from the estimates

$$
\begin{aligned}
& v\left(T_{0}, x\right)=a^{-1} \prod_{0}^{a} \psi(x) d x+ \\
& +\sum_{n=1}^{x} 2 a^{-1} \prod_{0}^{a} \psi(y) \cos \left(\frac{n \pi}{a} y\right) d y \cos \left(\frac{n \pi}{a} x\right) \exp \left(-\frac{n^{2} \pi^{2}}{a^{2}} T_{0}\right) \\
& \leqslant i-\varepsilon+2 a^{-1} \sum_{n=1}^{\infty} \exp \left(-\frac{n^{2} \pi^{2}}{a^{2}} T_{0}\right) \prod_{0}^{a}|\psi(y)| d y<\lambda-\frac{1}{2} \varepsilon .
\end{aligned}
$$

If the difference $\left|u\left(T_{0}, x\right)-w\left(T_{0}, x\right)\right|$ is less than $\frac{1}{2} \varepsilon$, then $u\left(T_{0}, x\right)<\lambda$ (because $\left.w\left(T_{0}, x\right)<\lambda-\frac{1}{2} \varepsilon\right)$. and the preliminary case in our proof shows that $u$ converges to zero. Hence it remains to estimate the difference $\left|u\left(T_{0}, x\right)-w\left(T_{0}, x\right)\right|$ for $x \in[0, a]$.

The function $z:=u-w$ is non-negative for $t \geqslant 0$ (because $u, w$ satisfy $(8)-(9)$ and $\varphi(x) \geqslant \psi(x))$, and satisfies

$$
z_{t}=z_{x x}+f(u)-f(w) \leqslant z_{x x}+c|u-w|=z_{x x}+c z,
$$

where $c:=\max _{[0,1]}\left|f^{\prime}(s)\right|$. Now, z may be estimated as a solution of the linear problem by

$$
\begin{aligned}
& |z(t, x)| \\
& \quad \leqslant \exp (c t)\left\{a^{-1} \int_{0}^{a}(\varphi(x)-\psi(x)) d x+\sum_{n=1}^{\infty} c_{n} \cos \left(\frac{n \pi}{a} x\right) \exp \left(-\frac{n^{2} \pi^{2}}{a^{2}} t\right)\right\},
\end{aligned}
$$


with

$$
c_{n}=2 a^{-1} \int_{0}^{a}(\varphi(x)-\psi(x)) \cos \left(\frac{n \pi}{a} x\right) d x,
$$

or, farther with the use of our assumptions by

$$
|z(t, x)| \leqslant \exp (c t)\left\{\gamma+2 \gamma \sum_{n=1}^{\infty} \exp \left(-\frac{n^{2} \pi^{2}}{a^{2}} t\right)\right\} .
$$

Since (by definitions) $\gamma \leqslant \delta(\varepsilon)$, we also have

$$
\left|z\left(T_{0}, x\right)\right| \leqslant \exp \left(c T_{0}\right) \delta(\varepsilon)\left\{1+2 \sum_{n=1}^{\infty} \exp \left(-\frac{n^{2} \pi^{2}}{a^{2}} T_{0}\right)\right\} \equiv \text { const } \delta(\varepsilon)
$$

and the value of $\delta(\varepsilon)$ will now be choosen equal to $\varepsilon /(2$ const $)$. The proof is finished.

Consider next the case where the nonlinear term $f$ satisfies

$$
\max _{[0,1]} f^{\prime}(s)<\frac{1}{2 a^{2}}
$$

THEOREM 4. Let the nonlinear term $f$ satisfy (20), (B) and the conditions $f^{\prime}\left(0^{+}\right)<0, f^{\prime}\left(1^{-}\right)<0$. Then the solution $u$ of $(8)-(10)$ tends to $0, \lambda$ or 1 as $t$ tends to infinity.

Proof. Differentiating (8) with respect to $x$ and using the symbols $v:=u_{x}, g(u):=f^{\prime}(u)$, we get:

$$
v_{t}=v_{x x}+g(u) v, \quad v(t, 0)=v(t, a)=0, \quad v(0, x)=\varphi^{\prime}(x) .
$$

Applying the transformation $V(t, x):=v(t, x) \exp (-k t)$ with $k:=\max _{[0,1]} g(s)$, we get for the function $V$ :

$$
V_{1}=V_{x x}+[g(u)-k] V
$$

with analogous conditions as for $v$. Now

$$
h^{u}(t, x):=g(u)-k \leqslant 0,
$$

so, applying Theorem 1 of [5] to any $u$ separately, we get for $V$ the following estimate independent of $u$ :

$$
|V(t, x)| \leqslant \underset{[0, a]}{2 \max }\left|\varphi^{\prime}(x)\right| \exp \left(-t / 2 a^{2}\right) .
$$

Hence, for the function $v$

(21) $\left|u_{x}(t, x)\right|=|v(t, x)|=|V(t, x) \exp (k t)| \leqslant 2 \max _{[0, a]}\left|\varphi^{\prime}(x)\right| \exp \left(k t-\frac{1}{2 a^{2}} t\right)$, 
which together with the estimate (20) ensures the convergence of $u_{x}$ to zero. Therefore, for sufficiently large $T>0$ one of the following possibilities holds: $u(T, x)>\lambda \quad($ then as a consequence of Theorem $1 u(t, x) \rightarrow 1$ as $t \rightarrow \infty)$,

$$
u(T, x)<\lambda \quad(\text { then } u(t, x) \rightarrow 0 \text { as } t \rightarrow \infty),
$$

for all $t>0 u(t, x)$ intersects $\lambda$ (then $u(t, x) \rightarrow \lambda$ as $t \rightarrow \infty$ ). or 1 .

6. Finally, consider the set of the solutions which do not converge to 0

Lemma 2. For symmetric $f$, weakly nonlinear;

$$
f(z)=-f(1-z), \quad f^{\prime}(z) \leqslant \beta<C^{-1} \quad \text { for } z \in[0,1],
$$

satisfying (B) and the initial condition satisfying

$$
0 \leqslant \varphi(x)=1-\varphi(a-x) \leqslant 1, \quad x \in[0, a]
$$

the solution $u$ of $(8)-(10)$ corresponding to $\varphi$ tends uniformly to $\lambda=\frac{1}{2}$.

Proof. It is easy to see that $(1-u(t, a-x))$ solves the same equation as $u(t, x)$, and moreover, with the same initial condition; hence by uniqueness

$$
0 \leqslant u(t, x)=1-u(t, a-x) \leqslant 1, \quad t \geqslant 0 .
$$

Consider now the equation for $u_{x}$

$$
u_{t x}=u_{x x x}+f^{\prime}(u) u_{x} .
$$

Multiplying the above by $u_{x}$ and integrating over $[0, a]$, we get

$$
\frac{d}{d t} \int_{0}^{a} u_{x}^{2} d x=-2 \int_{0}^{a} u_{x x}^{2} d x+2 \int_{0}^{a} f^{\prime}(u) u_{x}^{2} d x
$$

Now with the use of (22) and as a consequence of the Poincare inequality (6) we have

$$
\frac{d}{d t} \int_{0}^{a} u_{x}^{2} d x \leqslant 2\left(-C^{-1}+B\right) \int_{0}^{a} u_{x}^{2} d x,
$$

and hence $u_{x}$ tends to zero in $L^{2}(0, a)$ as $t$ tends to infinity. Using the Sobolev inequality [9]

$$
\underset{B>0}{\exists} \underset{w \in C^{1}([0, a])}{\forall}\|w\|_{C^{0}([0, a])}^{2} \leqslant B\left(\|w\|_{L^{2}}^{2}+\left\|w_{x}\right\|_{L^{2}}^{2}\right)
$$

for the function $w=v-\bar{v}(\bar{v}$ denotes the average of $v)$ and (7), we obtain the estimate

$$
\underset{k>0}{\exists} \underset{v \in C^{1}([0, a])}{\forall}\|v-\vec{v}\|_{C^{0}([0, a])}^{2} \leqslant k\left\|v_{x}\right\|_{L^{2}}^{2}
$$


which together with (24) gives

$$
\|u(t, \cdot)-\bar{u}(t)\|_{C^{0}([0 . a])}^{2} \leqslant k\left\|u_{x}(t, \cdot)\right\|_{L^{2}}^{2} \rightarrow 0, \quad t \rightarrow x .
$$

Since, (23), $\bar{u}(t)=\frac{1}{2}$, this gives the uniform convergence of $u$ to $\frac{1}{2}$.

In general case, using Ważewski's method, we may show that for arbitrary fixed function $f$ satisfying $f^{\prime}\left(0^{+}\right)<0, f^{\prime}\left(1^{-}\right)<0$ there exists continuum initial conditions $\varphi$ such that the solution of $(8)-(10)$ does not converge to 0 or 1 .

Definition. By a non-decreasing homotopy family (parameter $\mu$ ) of functions we mean every set of functions $\gamma:[0,1] \times[0, a] \rightarrow[0,1]$ of the arguments $(\mu, x)$ which satisfy:

if $\mu_{1} \geqslant \mu_{2}$, then

$$
\begin{gathered}
\gamma\left(\mu_{1}, x\right) \geqslant \gamma^{\prime}\left(\mu_{2}, x\right) \quad \text { for } x \in[0, a], \\
\gamma(0, x)=0, \quad \gamma(1, x)=1, \quad x \in[0, a], \\
\gamma_{x}(\mu, 0)=\gamma_{x}(\mu, 1)=0, \quad \mu \in[0,1],
\end{gathered}
$$

and the function $\mu \rightarrow \gamma(\mu, \cdot)$ is continuous in $C^{2+x}([0, a])$ for $\mu \in[0,1]$.

Let us consider an arbitrary fixed family. For small $\mu$ the solution of (8)-(9) with initial condition $\gamma(\mu, x)$ tends to 0 , for $\mu$ near 1 the solution tends to 1 . By Corollary 3 the set of $\mu$ for which the solutions tend to 0 coincides with an interval $\left[0, a_{1}\right)$ or $\left[0, a_{1}\right]$; analogously, the set of $\mu$ for which the solutions tend to 1 is an interval $\left(a_{2}, 1\right]$ or $\left[a_{2}, 1\right]$, where $a_{1} \leqslant a_{2}$. But both these intervals must be open from one side. If the first of them is closed, then arguing as in the second part of the proof of Theorem 3 (the trajectorie $: u(t, \cdot)_{i t \geqslant 0}, u(0, x)=\gamma\left(a_{1}, x\right)$ is compact in $C^{1}([0, a])$ and $u(t, x)$ tends to 0 pointwise as $t$ tends to infinity: hence for a sequence $\left\{t_{n}\right\}_{n \in N}$, $t_{n} \rightarrow \infty, u\left(t_{n}, \cdot\right)$ tends to 0 uniformly; the rest is a consequence of the continuous dependence of solutions on the initial condition as in Theorem 3) we see that for some $\mu>a_{1}$ the corresponding solution tends to zero. This contradicts the maximality of $a_{1}$. The solutions corresponding to the parameters $\mu$ from the remaining set $\left[a_{1}, a_{2}\right]$ must tend neither to 0 nor to 1 .

7. Some remarks concerning global in time existence of the solutions. As a consequence of our assumptions concerning $f$, when the values of $\varphi$ belong to $[0,1]$, the same holds true for $u$. As a standard consequence of Theorem 7.4 , p. 560 of [7], in any bounded in time cylinder there exists a $C^{1+\alpha / 2,2+\alpha}$ solution of (1)-(3). Additional smoothness assumed for $f$ in (B) ensures existence of the derivatives $D_{x}^{4} u, D_{1} D_{x}^{2} u$ for the solution of (8)-(10). There are several ways to show global in time a priori estimates for the derivatives $u_{x}, u_{x x}$ and $u_{\mathrm{t}}$. This is done, for example, in [4], Appendix B. Another approach is given in [3], Theorems 11 and 12, and in [2]. 
Acknowledgement. The author would like to express his thanks to Professor Andrzej Lasota for suggesting the problem and for many valuable discussions during the preparation of the work.

\section{References}

[1] R. F. V. Anderson, Stability of displacement clines arising in allospecies competition, J. Math. Biology 6 (1978), 131-144.

[2] N. Chafee, A stability analysis for a semilinear parabolic partial differential equation, J. Differ. Eqs 15 (1974). 522-540.

[3] Chzou Yui-lin, Boundary problems for nonlinear parabolic equations, Mat. Sborn. 47 (89) (1959), 431-483.

[4] E. Conway, D. Hoff, J. Smoller, Large time behatior of solutions of nonlinear reactiondiffusion equations, SIAM J. Appl. Math. 35 (1978), 1-16.

[5] Ju. Czeremnych, On asymptotic behaviour of solutions of parabolic equations, Izw. A.N. SSSR 23 (1959), 913-924.

[6] A. Friedman, Partial Differential Equations of Parabolic Type, Prentice Hall, Englewood Cliffs 1964.

[7] O. A. Ladyzenskaja, V. A. Solonnikov, N. N. Uralceva, Linear and Quasilinear Equations of Parabolic Type. Nauka, Moscow 1967.

[8] J. P. LaSalle, An incariance principle in the theory of stability, Int. Symp. Diff. Eqs Dyn. Syst., ed. J. K. Hale and J. P. LaSalle. Academic Press. 1967. 277-286.

[9] C. B. Morrey, Multiple Integrals in the Calculus of Variations, Springer Verlag. New York 1966.

[10] W. M. Poljakova, On stability of solutions of the heat equation (in Russian), D.A.N. SSSR 129 (1959). 1230-1233.

[11] J. Szarski, Differential Inequalities, PWN, Warsaw 1967.

INSTYTUT MATEMATYKI UNIWERSYTETL' ŚLASKIEGO, KATOWICE

INSTITUTE OF MATHEMATIC, SILESIAN UNIVERSITY, KATOWICE, POLAND 